http://dx.doi.org/10.21611/qirt.2004.b

\title{
Spatiotemporal Active Thermography
}

\author{
Jähne, B.
}

Interdisciplinary Center for Scientific Computing (IWR)

Germany

Standard active thermograph techniques such as lockinthermography or burst-phase-thermography apply only temporal varying patterns to the surface of the object to be investigated. As a consequence, the heat flow is basically directed perpendicularly to the surface. In contrast, spatially varying heat flow applied to surfaces causes also temperature differences parallel to the surface and thus is also sensitive to lateral conductivity changes. Applying spatially and temporally varying heat flows results in a powerful generalized technique for non-destructive and remote investigation of objects, which is called spatiotemporal active thermography (STAT). Some important basic features of this technique are worked out.

So far, active thermographic techniques have mainly been applied to static objects. There are, however, many interesting applications with moving parts to be investigated by active thermographic techniques. Especially demanding are processes with random motions. STAT is a powerful experimental technique even for such complex cases. This is demonstrated by the investigation of heat exchange across the aqueous viscous boundary at the wind-driven and wave-undulated ocean surface. 